Bond University

Research Repository

\title{
Is the "heart age" concept helpful or harmful compared to absolute cardiovascular disease risk? An experimental study
}

Bonner, Carissa; Jansen, Jesse; Newell, Ben R.; Irwig, Les; Teixeira-Pinto, Armando; Glasziou, Paul; Doust, Jenny; McKinn, Shannon; McCaffery, Kirsten

Published in:

Medical Decision Making

DOI:

10.1177/0272989X15597224

Licence:

Other

Link to output in Bond University research repository.

Recommended citation(APA):

Bonner, C., Jansen, J., Newell, B. R., Irwig, L., Teixeira-Pinto, A., Glasziou, P., Doust, J., McKinn, S., \& McCaffery, K. (2015). Is the "heart age" concept helpful or harmful compared to absolute cardiovascular disease risk? An experimental study. Medical Decision Making, 35(8), 967-978.

https://doi.org/10.1177/0272989X15597224

\section{General rights}

Copyright and moral rights for the publications made accessible in the public portal are retained by the authors and/or other copyright owners and it is a condition of accessing publications that users recognise and abide by the legal requirements associated with these rights.

For more information, or if you believe that this document breaches copyright, please contact the Bond University research repository coordinator. 
Title: Is the 'heart age' concept helpful or harmful compared to absolute cardiovascular disease risk? An experimental study

Running head: Heart age versus absolute CVD risk

Authors: Ms Carissa Bonner, $\mathrm{MPH}^{1,2}$, Dr Jesse Jansen, $\mathrm{PhD}^{1,2}, \mathrm{~A} / \operatorname{Prof}$ Ben $\mathrm{R}$ Newell, $\mathrm{PhD}^{3}$, Prof Les Irwig, MBBS, PhD ${ }^{1}$, Dr Armando Teixeira-Pinto, $\mathrm{PhD}^{1}$, Prof Paul Glasziou, MBBS, PhD ${ }^{1,4}$, Prof Jenny Doust, MBBS, PhD ${ }^{1,4}$, Miss Shannon McKinn, MIPH ${ }^{1,2}, \mathrm{~A} /$ Prof Kirsten McCaffery, PhD ${ }^{1,2}$

1. Screening and Test Evaluation Program (STEP), Sydney School of Public Health, The University of Sydney, NSW 2006, Australia.

2. Centre for Medical Psychology and Evidence-based Decision-making (CeMPED), The University of Sydney, NSW 2006, Australia

3. School of Psychology, University of New South Wales, NSW 2052, Australia.

4. Faculty of Health Sciences and Medicine, Bond University, QLD 4229, Australia.

Correspondence to: A/Prof Kirsten McCaffery, kirsten.mccaffery@sydney.edu.au

Word count: 4571

Financial support for this study was provided in part by a Vanguard Grant (No. 100225) from the National Heart Foundation of Australia, and in part by a Program Grant awarded to the Screening and Test Evaluation Program (STEP; No. 633003) from the National Health and Medical Research Council (NHMRC). The funding agreements ensured the authors' independence in designing the study, interpreting the data, writing, and publishing the report. 
The final, definitive version of this paper has been published in Medical Decision Making 35(8),

2015 published by SAGE Publishing, All rights reserved. DOI: 10.1177/0272989X15597224 


\section{ABSTRACT}

Background: Cardiovascular disease (CVD) prevention guidelines are generally based on the absolute risk of a CVD event, but there is increasing interest in using 'heart age' to motivate lifestyle change when absolute risk is low. Previous studies have not compared heart age to 5-year absolute risk, or investigated the impact of younger heart age, graphical format and numeracy.

Objective: Compare heart age versus 5-year absolute risk on psychological/behavioural outcomes. Design: 2 (heart age, absolute risk) x 3 (text only, bar graph, line graph) experiment.

Setting: Online.

Participants: 570 Australians aged 45-64 years, not taking CVD-related medication.

Intervention: CVD risk assessment.

Measurements: Intention to change lifestyle, recall, risk perception, emotional response, perceived credibility, lifestyle behaviours after 2 weeks.

Results: Most participants had lifestyle risk factors (95\%) but low 5-year absolute risk (94\%). Heart age did not improve lifestyle intentions/behaviours compared to absolute risk, was more often interpreted as a higher risk category by low risk participants ( $47 \%$ vs $23 \%$ ), and decreased perceived credibility and positive emotional response. Overall, correct recall dropped from $65 \%$ to $24 \%$ after 2 weeks, with heart age recalled better than absolute risk at 2 weeks ( $32 \%$ vs 16\%). These results were found across younger/older heart age result, graphical format and numeracy.

Limitations: Communicating CVD risk in a consultation rather than online may produce different results.

Conclusions: There is no evidence that heart age motivates lifestyle change more than 5 -year absolute risk in individuals with low CVD risk. Five-year absolute risk may be a better way to explain CVD risk, since it is more credible, does not inflate risk perception, and is consistent with clinical guidelines that base lifestyle and medication recommendations on absolute risk. 


\section{INTRODUCTION}

Clinical guidelines for cardiovascular disease (CVD) prevention generally base medication recommendations on the absolute risk of a CVD event rather than managing individual risk factors. ${ }^{1}$ This is expressed as the short-term percentage risk of a cardiovascular event, usually over a 5 or 10 year timeframe. However, adherence to these guidelines is suboptimal around the world, ${ }^{2}$ and clinicians report difficulties communicating absolute risk to patients. ${ }^{3}$ Research has established that percentages are poorly understood by both patients and clinicians. ${ }^{4,5}$ Moreover, the absolute risk concept may also undermine lifestyle change messages for patients with low short-term CVD risk but elevated risk factors that increase their lifetime risk. ${ }^{6,7}$ To address concerns that the absolute risk approach may miss opportunities to intervene before absolute risk becomes high, recent clinical guidelines in the UK advocate the use of 'heart age' to communicate lifetime risk and motivate prevention measures when absolute risk is still low. ${ }^{8}$

Heart age is calculated by comparing an individual's current absolute risk to the age at which they would reach that absolute risk if they had 'ideal' risk factors (e.g. non-smoker, systolic blood pressure $120 \mathrm{mmHg}$, total/HDL cholesterol ratio 4). ${ }^{9}$ Older heart age than current age indicates elevated but modifiable risk, even if the short-term absolute risk of a CVD event is low (see Box 1). Younger heart age indicates that current risk factors are even lower than 'ideal' levels. The New Zealand Heart Foundation introduced an online calculator to promote the use of absolute risk guidelines in 2010 including both short-term (5-year) absolute risk and heart age in a line graph format, showing how CVD risk will increase over time if current risk factors remain the same compared to if they were ideal. ${ }^{9} \mathrm{~A}$ pre-post study suggested that this tool may increase clinician understanding and confidence regarding the use of absolute risk, but patient outcomes were not assessed. ${ }^{10}$ Recent clinical guidelines in the UK specifically advocate using heart age for lower risk 
patients and even include medication recommendations based on heart age. ${ }^{8}$ The rationale for this change is that heart age reflects lifetime risk, and could potentially motivate patients with low short term risk to improve their lifestyle before their risk becomes high. However, there is little evidence that heart age is more effective than other risk formats, as previous studies have not directly compared it to 5 year absolute risk, and have not investigated the impact of being told younger compared to older heart age or graphical format.

Qualitative studies suggest that patients may prefer heart age over other formats but raise concerns about its negative emotional impact. A focus group study using hypothetical risk found that patients liked heart age but were concerned it may frighten people if higher than their current age. ${ }^{11} \mathrm{~A}$ 'think aloud' study where patients used online CVD risk tools to calculate their actual heart age found that patients often misunderstand and discredit the results of heart age calculators, but they were prompted to consider lifestyle changes regardless of whether they believed the result or had a negative reaction to older heart age. ${ }^{12}$ Quantitative studies suggest that heart age may be effective for motivating lifestyle change and reducing CVD risk factors, but there is little direct evidence that it is more effective than alternative risk formats. An experimental study found that heart age improved understanding of risk compared to 10-year absolute risk and had more emotional impact for younger people at higher risk. Emotional impact also mediated the relationship between risk level and intention to change lifestyle for both formats. ${ }^{13}$ Comparison of heart age to 5 -year absolute risk has not been addressed in previous studies, but a review of hypothetical absolute CVD risk formats suggests that shorter time frames ( $<10$ years) may improve the accuracy of risk perception and increase intentions to change behaviour compared to longer time frames (10 years or more). ${ }^{14}$ Clinical trials have found that risk communication including heart age, and the similar concept of lung age, can motivate behaviour change and improve risk factors, but these age-related formats were not directly compared to alternative risk formats. ${ }^{15,16}$ 
There is no consensus on the 'best' way to present quantitative health risks in the broader risk communication literature, but the review of CVD risk formats mentioned above recommends graphical presentation. ${ }^{14}$ Best practice guidelines suggest that graphical formats have mixed effects compared to text-only presentations, and their benefit may depend on individual differences in numeracy and literacy. ${ }^{17,18}$ However, there is some research to suggest that bar graphs and frequency-based icon arrays can be beneficial for understanding health risks, particularly for people with lower numeracy. ${ }^{19}$ This effect may be due to reducing various cognitive errors in the interpretations of textual expressions of risk, such as denominator neglect. ${ }^{18,20}$ Since heart age is based on a comparison to ideal risk but does not directly relate to frequencies, bar graphs comparing actual versus ideal risk assessment results were used in this study, as an alternative to text only and line graph formats.

Previous research has not investigated the effect of heart age compared to 5-year absolute risk, which is currently used in Australian and New Zealand guidelines, charts, and online tools. ${ }^{9}{ }^{21}$ Nor have there been any studies investigating the effect of communicating younger heart age, or presenting heart age in different graphical formats. This study aimed to address these gaps in the literature by experimentally testing the effect of heart age on psychological and behavioural outcomes compared to 5-year absolute risk_in low (i.e., 5-year absolute risk of a CVD event $<10 \%$ ), to moderate risk (10-15\% AR) patients, according to the Australian guidelines.

\section{METHOD}

\section{Design}

A $2 \times 3$ factorial design was used to investigate the effect of CVD risk format (5-year absolute risk, heart age) and different graphical formats (text only, text + bar graph, text + line graph) on 
psychological and behavioural outcomes. The full study protocol is provided in the supplementary material, including examples of all presented CVD risk formats. This paper focuses on the effect of heart age compared to 5-year absolute risk on the main outcomes of interest in previous heart age literature (recall, perceived risk category, emotional response, perceived credibility, intention to change lifestyle, and self-reported behaviour change). See Figure 1. Additional psychological outcomes were assessed to explore possible mechanisms for any effect of heart age on lifestyle intention (alternative risk perception measures: numerical risk, verbal risk, comparative risk, feeling of risk; ${ }^{22}$ and illness representation measures: worry about heart disease, perceived control and timeline of heart disease, subjective understanding of heart disease ${ }^{23,24}$ ). However, since the hypothesised effect of heart age on lifestyle intentions was not found, main effects and interactions between risk and graphical formats for these additional outcomes are reported as exploratory analyses in the supplementary material.

[INSERT FIGURE 1]

\section{Sample size}

The a priori sample size calculation indicated that 85 participants per group, totalling 510 participants, would provide $90 \%$ power to detect a moderate effect size of $d=0.5$ (standardised difference) in the primary outcome of intention to change lifestyle as measured immediately post intervention, assuming a two-sided alpha of 0.05 . We aimed to recruit $20 \%$ more cases to account for potential dropout at two week follow-up.

\section{Recruitment}


A national Australian sample was recruited through a market research company (SSI: Survey Sampling International) in 2014. Sampling quotas were set to ensure equal participant numbers by gender and 5 year age group, since these are important determinants of CVD risk and we aimed to recruit participants with both younger and older heart age results at a range of ages. The eligibility criteria were being aged 45-64 years, not currently taking blood pressure or cholesterol-lowering medication, non-diabetic, not already known to be at high risk of CVD based on criteria in the Australian guidelines, and being able to read health-related information in English without help most of the time. Participants were recruited via email or by visiting the SSI website independently. They received points for participating in the study, which they could redeem for entry into prize draws through SSI's online system.

\section{Materials}

Participants completed a CVD risk assessment based on Australian absolute risk guidelines and the New Zealand Heart Foundation's approach to calculating heart age, using the 5-year Framingham risk equation ${ }^{9,21,25}$ and determining heart age by comparing a participant's current absolute risk to the age at which they would reach that absolute risk if they had 'ideal' risk factors (i.e. non-smoker, systolic blood pressure $120 \mathrm{mmHg}$, total/HDL cholesterol ratio 4). If blood pressure or cholesterol were not known the average by age and gender was used based on data from non-diabetic participants who were not taking blood pressure or cholesterol-lowering medication in the AusDiab study: a longitudinal study of 11,000 people investigating diabetes prevalence in Australia. ${ }^{26}$ If participants indicated that a health professional had told them their blood pressure and/or cholesterol was raised, one standard deviation was added to the average for their age and gender. An example of the text for different risk formats is provided in Box 1, with additional examples in Box 2, and graphical presentation formats provided in the supplementary material. 


\section{Box 1. Example of randomised risk formats}

A 50 year old male smoker with blood pressure $=130 / 80 \mathrm{mmHg}$ and cholesterol ratio $=4$ would be compared to a 50 year old male with 'ideal' risk factors (non-smoker, blood pressure $=$ $120 / 80 \mathrm{mmHg}$, cholesterol ratio $=4$ ), and receive his results in one of the following formats, depending on which group he was randomised to (note: non-smokers did not receive information about quitting smoking; and heart age was set to a maximum of 74 , in line with the Framingham model used in Australian guidelines and the New Zealand heart age tool).

\section{Absolute risk group:}

"Your risk of having a heart attack or stroke in the next 5 years is $8 \%$. If you stopped smoking and had lower blood pressure and cholesterol, your risk would be $3 \%$. You can reduce your blood pressure and cholesterol by quitting smoking, improving your diet and increasing physical activity."

\section{Heart age group:}

"Your heart age is 66, 16 years older than you. If you stopped smoking and had lower blood pressure and cholesterol, your heart age would be 50 , the same as your current age. You can reduce your blood pressure and cholesterol by quitting smoking, improving your diet and increasing physical activity." 


\section{Box 2. Example of text results}

All participants received their results in text format, with the exact wording depending on their randomised risk format (percentage risk or heart age), whether their calculated heart age result was older, the same or younger than their current age, and whether they were a smoker or not.

Older heart age result than current age, percentage risk format, non-smoker:

"Your risk of having a heart attack or stroke in the next 5 years is $5 \%$. If you had lower blood pressure and cholesterol, your risk would be $2 \%$. You can reduce your blood pressure and cholesterol by improving your diet and increasing physical activity."

\section{Older heart age result than current age, percentage risk format, smoker:}

"Your risk of having a heart attack or stroke in the next 5 years is $17 \%$. If you stopped smoking and had lower blood pressure and cholesterol, your risk would be $5 \%$. You can reduce your blood pressure and cholesterol by quitting smoking, improving your diet and increasing physical activity."

\section{Same heart age result as current age, heart age format:}

"Your heart age is 48 , the same as your current age. Congratulations, this is the ideal result for your age and gender."

\section{Younger heart age result than current age, heart age format:}

"Your heart age is 45, 3 years younger than you. Congratulations, this is even better than the ideal result for your age and gender." 


\section{Outcome measures}

The question format and response options for all measures are provided in the supplementary material. Demographic, clinical and lifestyle risk factors were assessed pre-intervention. Numeracy was also assessed at this point using the validated Subjective Numeracy Scale. ${ }^{27}$

Psychological measures were assessed immediately post-intervention:

a) The primary outcome was intention to change lifestyle (improve diet, increase physical activity, stop smoking if applicable). This was assessed using a 3 item Likert scale from the Theory of Planned Behavior for each lifestyle aspect, a well-established approach in the behavioural science literature. ${ }^{28}$ For example, for physical activity, participants rated the following statements from 1=strongly disagree to 7=strongly agree: I expect to do more physical activity in the next 2 weeks; I want to do more physical activity in the next 2 weeks; I intend to do more physical activity in the next 2 weeks. Average scores for each lifestyle aspect were calculated as well as the average across applicable lifestyle aspects ( 6 items for non-smokers, 9 items for smokers). ${ }^{28,29}$

b) Recall of heart age or absolute risk result was assessed as correct (i.e. the exact right answer) or incorrect based on open numerical responses (and re-assessed at 2-week follow-up).

c) Risk perception was assessed as the perceived risk category that the result indicated. In line with the Australian guidelines, low was defined as a 5 -year absolute risk of a CVD event $<10 \%$, moderate is $10-15 \%$ and high $>15 \%$ AR. These categories were not defined for participants in the study materials, as we were interested in the participants' own impressions of their risk level, not their understanding of the categories as defined by the Australian guidelines.

d) Emotional response was assessed using a 6 item Likert scale (from $0=$ "none of this feeling" to $10="$ a lot of this feeling"), with positive (hopeful, optimistic, enthusiastic; $\alpha=.81$ ) and negative (afraid, anxious, worried; $\alpha=.85$ ) subscales scored based on the average of the relevant items, used in previous research on the role of affect in health risk information seeking. ${ }^{30}$ 
e) Perceived credibility was assessed with a 4 item Likert scale (from 1="completely disagree" to $7="$ completely agree") scored as the average of the items (I felt that the numbers received were "my numbers"; I found the results to be written personally for me; I felt that the information was relevant to me; I felt that the information was designed specifically for me; $\alpha=.89$ ), used in previous research on the personal relevance and believability of online health risk assessments. ${ }^{31}$

Behavioural/lifestyle measures were assessed pre- and post-intervention, and at 2 week follow-up: f) Information seeking was assessed by providing three links in randomised order at the end of the post-intervention questionnaire. Clicking on either of the two CVD-related links (to information about CVD risk and lifestyle on the Australian Heart Foundation website) was coded as a positive response; clicking on a link to Google or closing the browser was coded as a negative response, in line with the methods used in previous studies investigating online risk information seeking. ${ }^{32}$ g) Intention to see a GP was assessed post-intervention using a 3 item Likert scale for intention as per the primary outcome, based on the average rating for the following statements from 1=strongly disagree to 7=strongly agree: I expect to discuss my risk of heart disease with a doctor in the next 2 weeks; I want to discuss my risk of heart disease with a doctor in the next 2 weeks; I intend to discuss my risk of heart disease with a doctor in the next 2 weeks. ${ }^{29}$ Participants also reported whether they had seen or made an appointment to see their GP at 2 week follow-up (response options: yes or no).

h) Smoking status was assessed pre-intervention and at 2 week follow-up with the question "Do you currently smoke cigarettes?" (response options: yes or no).

i) Physical activity was assessed pre-intervention and at 2 week follow-up using the 2Q-PA scale for moderate and vigorous physical activity, with a time scale of "in the last week" added. Adequate physical activity was defined as $\geq 3$ vigorous sessions/week, or $\geq 5$ moderate sessions/week, or 1-2 vigorous sessions/week plus 3-4 moderate sessions/week. ${ }^{33}$ 
j) Diet was assessed pre-intervention and at 2 week follow-up using questions from a previous study on fruit and vegetable intake, modified to be consistent with Australian guidelines and the physical activity response options. Adequate diet was defined as having at least 2 servings of fruit and 5 servings of vegetables per day in the last week. ${ }^{34}$

k) Body mass index was calculated using self-reported height and weight measures pre-intervention with options to provide this in either metric or imperial units. Clinically impossible values were recorded as missing.

\section{Analysis}

Continuous variables were described as mean (SD), or median (IQR: interquartile range) if the distributions were asymmetric. Categorical variables were summarised with percentages. The MannWhitney test (non-parametric) was used to compare groups across continuous outcomes, and the Chi-square test was applied to categorical outcomes. We further explored the dependence of the results on participants' calculated heart age result (younger/same versus older than current age) and numeracy by testing an interaction using logistic and quantile (for the median) regressions for categorical and continuous outcomes, respectively. Finally, we compared the differences between the group arms for the primary outcomes, adjusted for characteristics that were slightly unbalanced across the groups after randomisation, namely, gender, BMI, blood pressure assessed in the last year and having been told the cholesterol was high. For the categorical outcomes we performed the adjustment using logistic or multinomial regression and for the continuous outcomes we used quantile regressions.

The significance level for all tests was set at 0.05. 
Participants were recruited via email or by visiting the SSI website independently, and quotas were set to achieve equal participant numbers in each age/gender category, so an exact response rate cannot be calculated. Of 3027 people who accessed the website during the recruitment period 141 did not consent to participate, 498 did not meet the eligibility criteria, and 1783 did not complete the questionnaire before their age/gender quota was full and their eligibility data were not recorded. Recruitment was closed after 605 participants had been randomised and completed the whole questionnaire. Of these 605 participants 35 were excluded from the analyses because they specified their blood pressure and/or cholesterol values as zero, which meant the Framingham risk equation could not be calculated and they could not be shown their allocated CVD risk format. The remaining 570 participants were included in the analysis for post-intervention outcomes, of which 469 participants (82\%) completed the follow-up questionnaire for 2 week outcomes. See Figure 1. Baseline characteristics and calculated risk results for those included in the post-intervention analyses are displayed in Table 1; psychological and behavioural outcomes are displayed in Table 2 and Figures 2 and 3.

\section{Participant characteristics}

The randomised groups were similar in terms of CVD risk factors with most participants having lifestyle risk factors (95\% had inadequate physical activity, inadequate diet, higher than normal body mass index and/or were current smokers) but low short-term absolute risk of a CVD event (94\% had 5 year absolute risk $<10 \%$ ). Calculated heart age was on average 3 years older than current age ( 57 versus 54 years) with $63 \%$ of participants across randomised groups having a higher heart age than current age, $1 \%$ having the same heart age as their current age, and $36 \%$ having a lower heart age than current age. 


\section{Primary outcome}

For intention to change lifestyle (diet, physical activity, smoking, and the average of these), there were no significant differences between the absolute risk and heart age groups (see Table 2). There were no significant interactions with numeracy, i.e., there was no evidence that differences between the groups were affected by participants' numeracy. These results did not change after adjustment for gender, BMI, blood pressure assessed in the last year or having been told the cholesterol was high.

\section{Secondary outcomes}

Several differences between the absolute risk and heart age groups were found for secondary outcomes, and there were additional subgroup differences when participants with a younger/same calculated heart age were analysed separately from participants with an older calculated heart age.

\section{Recall}

The majority of participants (65\%) correctly recalled their risk result immediately post-intervention, but this dropped to $24 \%$ after 2 weeks. Participants in the heart age group were more likely to correctly recall their risk result after 2 weeks compared to those in the absolute risk group across younger and older heart age results ( $32 \%$ versus $16 \% ; p<0.001 ;)$. For participants with a younger or same calculated heart age compared to current age, those who received their result in heart age format were more likely to correctly recall their result post-intervention compared to those who received an absolute risk format ( $80 \%$ versus $63 \%, p=0.009$ ). The risk format did not make a difference in post-intervention recall amongst participants with a higher calculated heart age $(61 \%$ for both formats, $p>0.999)$. As a sensitivity analysis we tested whether there was any impact on the 
recall results when a recalling margin of error was allowed for (ranging from 0.05-0.2 standard deviations). The results were similar, with recall better in the heart age condition. In fact the heart age format was further favoured since the error was much smaller in the heart age condition.

\section{Risk perception}

Most participants (94\%) were in the low risk category according to Australian guidelines (5-year absolute risk of a CVD event $<10 \%),{ }^{21}$ but only $53 \%$ of participants in the heart age group perceived the result as indicating low risk of having a heart attack or stroke compared to $77 \%$ in the absolute risk group ( $p<0.001$ ). Of the $94 \%$ low risk participants who received their risk result in absolute risk format ( $n=266), 78 \%$ perceived their result to be indicating low risk, $20 \%$ perceived it to be moderate risk, and $2 \%$ perceived it to be high risk. Of the low risk participants who received their risk result in heart age format ( $n=269$ ) $56 \%$ perceived their result to be indicating low risk, $40 \%$ perceived it to be moderate risk, and $4 \%$ perceived it to be high risk (see Figure 2). For those with moderate/high risk the effect of heart age is unclear. There were no significant differences between risk perception and actual risk by format $(p=0.071)$, however, the small number of cases (with only 1 high risk participant who was randomized to the absolute risk arm) limit the conclusions for this group.

\section{Emotional response}

Participants in the heart age group had a less positive emotional response (hopeful, optimistic, enthusiastic) to the risk result compared to participants in the absolute risk group (median 5.0 versus $6.0 ; p<0.001$ ). Heart age also increased negative emotional response (afraid, anxious, worried) compared to absolute risk, but this difference was not statistically significant (median 3.0 versus 2.3; $p=0.40$; see Figure 3 ). 


\section{Perceived credibility}

Perceived credibility of the risk result (i.e. whether the results were believed/personally relevant) was lower for participants in the heart age group compared to participants in the absolute risk group (median 4.8 versus 4.3; $p<0.001$; see Figure 3 ).

\section{Behavioural outcomes}

No risk format effects were found for behavioural outcomes immediately post-intervention (intention to see a GP, information seeking behaviour) or at 2 week follow-up (percentage adequate diet, adequate physical activity, smokers, GP appointment for CVD risk assessment).

[INSERT TABLES 1-2 AND FIGURES 2-3]

\section{DISCUSSION}

The results of this study indicate that online assessment of heart age is no more effective than 5year absolute risk for increasing lifestyle change intentions and behaviour, in a general population sample with lifestyle risk factors but mostly low short-term risk of a CVD event (mean 5-year absolute risk $<5 \%$ in both groups). The sample size was sufficient to assess this as the primary outcome, so the result is unlikely to be due to a lack of power, and the sample characteristics reflected the target group for heart age communication in recent clinical guidelines advocating the use of heart age. ${ }^{8}$ Compared to 5 -year absolute risk, heart age was more likely to be interpreted as moderate/high risk by low risk participants, decreased positive emotional response to the results, and decreased the perceived credibility of the risk assessment; however, heart age was more likely 
to be accurately recalled after 2 weeks. These effects were found across younger and older heart age results and numeracy.

Our findings are in line with a recent experimental study that found adding heart age to salient absolute risk formats had no effect on lifestyle intentions ${ }^{35}$ and contradict suggestions that it is more motivating than absolute risk in individuals with low CVD risk. ${ }^{8,9,13}$ Five-year absolute risk is by definition a smaller number than 10-year absolute risk, and people have a tendency to ignore the base rate for probabilistic information, including the time frame of health statistics. ${ }^{36}$ Moreover, people are so familiar with their own age that it stands as an available benchmark to compare heart age to. Theoretically, heart age could therefore be expected to have even more impact compared to 5-year rather than 10-year absolute risk, since the number indicating 'risk' is smaller when a 5-year time frame is used. However, our findings suggest that the prospect of a CVD event in the short-term has as much impact on lifestyle intention as the heart age format. This is supported by a previous review of absolute CVD risk formats that found shorter time frames ( $<10$ years) increase intentions to change behaviour compared to longer time frames (10 years or more) in hypothetical studies. ${ }^{14}$ The issue of low credibility identified in our previous qualitative study of online heart age calculators was also confirmed in this study: patients discredit the results of online heart age calculators, and perceive heart age as less credible than absolute risk. ${ }^{12}$ The finding that heart age has more emotional impact and is more memorable than absolute risk supports previous quantitative and qualitative studies, ${ }^{11-13}$ but this did not translate into greater intentions to change lifestyle. This is in contrast to a recent Spanish trial (published after this study was conducted) that found greater reductions in lifestyle and clinical risk factors for CVD after 12 months when patients were shown an interactive heart age tool by their clinician compared to usual care or communicating 10 -year absolute risk in verbal format. ${ }^{37}$ The discrepancy between these findings may be explained by the presentation formats used: the heart age group in the Spanish trial viewed an interactive, computerbased heart age tool demonstrating the effect of risk factor modification during a clinical 
consultation as well as the verbal format that the absolute risk group received (personal communication with corresponding author), so the effect may be attributed to a more compelling visual format rather than heart age per se. In the current study, the heart age and absolute risk groups viewed equivalent verbal and visual formats to enable a direct comparison (see supplementary material). Alternatively, 5 -year absolute risk and heart age may both be superior to 10-year absolute risk, but no different from each other, in terms of motivation to change lifestyle. The lack of impact of numeracy in this study contributes to the mixed findings in the broader risk communication literature. ${ }^{18}$

The main clinical implication of this study is that advocating heart age as a more effective risk communication format than absolute risk may be premature. Providing a 5-year absolute risk format may be a better way to explain risk to patients, since it is more credible, does not artificially inflate risk perception, and is consistent with clinical guidelines that base lifestyle and medication recommendations on absolute risk thresholds. ${ }^{1}$ On the other hand, heart age is recalled better, and assessment by a clinician may ameliorate the reduced credibility for heart age when provided via an online calculator. Assessment of heart age in a clinical consultation could also provide an opportunity to correct inaccurate risk perception and clearly explain the difference between short term risk (e.g. 5-year risk of a CVD event) and lifetime risk (reflected by the heart age concept). An additional benefit could be improved use of the absolute risk guidelines by GPs, if communicating heart age rather than absolute risk drives greater confidence in using the guidelines. ${ }^{10}$ Although these guidelines are targeted at clinicians, the online CVD risk and heart age calculators that support them are publicly available (www.jbs3risk.com; www.knowyournumbers.co.nz). Such health risk calculators are widely available online and used by consumers without clinician involvement, ${ }^{38}$ so effective CVD risk communication formats for both clinical and online settings are needed. 
The strengths of this study include a rigorous experimental design specifically testing heart age alone against 5-year absolute risk alone using equivalent verbal and visual formats, inclusion of a follow-up time point to assess behaviour change as well as psychological outcomes, and a national sample in the target group for heart age communication (i.e. lifestyle risk factors but low short-term risk of a CVD event). The limitations include the use of a static online format, which may not be as impactful as an interactive website or clinician communication during a consultation, the use of information seeking behaviour as a proxy for actual behaviour, the fact that we only included computer-literate participants and self-reported outcomes, and the need to exclude some participants due to inaccurate self-reported risk factor data.

The findings of this study could be extended by comparing the effect of communicating heart age versus 5-year absolute risk in clinical consultations using equivalent verbal and visual formats. Clinician involvement in communicating heart age may prevent the reduction in credibility and inaccurate risk perception found in this study, and exploratory analyses of risk and illness perceptions suggest that heart age may be useful in specific situations (see supplementary material). Further research is required to better understand the impact of the different formats on participants at moderate and high CVD risk since the majority of participants in this study were at low risk. Research should also confirm whether: 1) communicating text-based heart age or adding a bar graph to absolute risk is beneficial when GPs want to increase risk and illness perceptions for patients with low short-term absolute risk but lifestyle risk factors; 2) communicating younger heart age is beneficial for reducing risk perceptions amongst anxious low risk patients, without demotivating good lifestyle behaviours; and 3) our findings hold when using different time horizons. Alternative ways to explain the difference between short-term and lifetime risk, and the relationship between heart age and absolute risk, could also be explored. Since the benefits of lifestyle are not restricted to CVD, less specific age-related formats such as 'biological age' or 'real age' may also be helpful for motivating lifestyle change without inflating CVD risk perception. 
This study found that heart age was more memorable and had more emotional impact than 5-year absolute risk, but there was no benefit with respect to changing lifestyle intentions or behaviour. Communicating 5-year absolute risk may be the better approach, since it is more credible, avoids inflating risk perception, and is consistent with clinical guidelines that base lifestyle and medication recommendations on absolute risk thresholds. 


\section{Acknowledgements}

The authors would like to thank the participants, Nasser Bin Dhim for programming the experiment, and Dora Eflin for assistance with recruitment.

\section{Author contributions}

Author contribution were as follows: study design (CB, JJ, BN, LI , AT, PG, JD, KM), recruitment (CB, $S M)$, data collection $(C B)$, analysis (CB, AT), interpretation (CB, JJ, BN, LI, AT, PG, JD, SM, KM), drafting (CB) and revising the manuscript (CB, JJ, BN, LI, AT, PG, JD, SM, KM). All authors approved the final version of the manuscript.

\section{Ethical approval}

Ethical approval for the study was obtained through the Human Research Ethics Committee of The University of Sydney (Protocol No. 2013/914). Participation was anonymous.

\section{Competing interests}

The authors declare they have no conflicts of interest. 


\section{REFERENCES}

1. Ferket BS, Colkesen EB, Visser JJ, Spronk S, Kraaijenhagen RA, Steyerberg EW, et al. Systematic review of guidelines on cardiovascular risk assessment: Which recommendations should clinicians follow for a cardiovascular health check? Arch Intern Med. 2010;170(1):27-40.

2. Sposito AC, Ramires JAF, Jukema JW, Molina JC, Silva PMD, Ghadanfar MM, et al. Physicians' attitudes and adherence to use of risk scores for primary prevention of cardiovascular disease: Cross-sectional survey in three world regions. Curr Med Res Opin. 2009;25(5):1171-78.

3. Hobbs FDR, Jukema JW, Da Silva PM, McCormack T, Catapano AL. Barriers to cardiovascular disease risk scoring and primary prevention in Europe. QJM 2010;103(10):727-39.

4. Akl EA, Oxman AD, Herrin J, Vist GE, Terrenato I, Sperati F, et al. Using alternative statistical formats for presenting risks and risk reductions. Cochrane Database Syst Rev. 2011;(3):CD006776.

5. Visschers VHM, Meertens RM, Passchier WWF, de Vries NNK. Probability information in risk communication: A review of the research literature. Risk Anal. 2009;29(2):267-87.

6. Bonner C, Jansen J, McKinn S, Irwig L, Doust J, Glasziou P, et al. General practitioners' use of different cardiovascular risk assessment strategies: A qualitative study. Med J Aust. 2013;199(7):485-89.

7. Bonner C, Jansen J, McKinn S, Irwig L, Doust J, Glasziou P, McCaffery K. Communicating cardiovascular disease risk: An interview study of general practitioners' use of absolute risk within tailored communication strategies. BMC Fam Pract. 2014;15:106.

8. Joint British Societies Board. Joint British Societies' consensus recommendations for the prevention of cardiovascular disease (JBS3). Heart. 2014;100:1-67.

9. Wells S, Kerr A, Eadie S, Wiltshire C, Jackson R. 'Your Heart Forecast': A new approach for describing and communicating cardiovascular risk? Heart. 2010;96(9):708-13. 
10. Wells S, Kerr A, Broadbent E, MacKenzie C, Cole K, McLachlan A. Does Your Heart Forecast help practitioner understanding and confidence with cardiovascular disease risk communication? J Prim Health Care. 2011;3(1):4-9.

11. Goldman RE, Parker DR, Eaton CB, Borkan JM, Gramling R, Cover RT, et al. Patients' perceptions of cholesterol, cardiovascular disease risk, and risk communication strategies. Ann Fam Med. $2006 ; 4(3): 205-12$

12. Bonner C, Jansen J, Newell BR, Irwig L, Glasziou P, Doust J, et al. I don't believe it, but I'd better do something about it: Patient experiences of online heart age risk calculators. J Med Internet Res. 2014;16(4).

13. Soureti A, Hurling R, Murray P, van Mechelen W, Cobain M. Evaluation of a cardiovascular disease risk assessment tool for the promotion of healthier lifestyles. Eur J Cardiovasc Prev Rehabil. 2010;17(5):519-23.

14. Waldron CA, van der Weijden T, Ludt S, Gallacher J, Elwyn G. What are effective strategies to communicate cardiovascular risk information to patients? A systematic review. Patient Educ Couns. 2011;82(2):169-81.

15. Grover SA, Lowensteyn I, Joseph L, Kaouache M, Marchand S, Coupal L, et al. Patient knowledge of coronary risk profile improves the effectiveness of dyslipidemia therapy: The CHECK-UP study: A randomized controlled trial. Arch Intern Med. 2007;167(21):2296-303.

16. Parkes G, Greenhalgh T, Griffin M, Dent R. Effect on smoking quit rate of telling patients their lung age: The Step2quit randomised controlled trial. BMJ. 2008;336(7644):598-600.

17. Lipkus IM. Numeric, verbal, and visual formats of conveying health risk: Suggested best practices and future recommendations. Med Decis Making. 2007;27:696-713.

18. Trevena LJ, Zikmund-Fisher BJ, Edwards A, Gaissmaier W, Galesic M, Han PKJ, et al. Presenting quantitative information about decision outcomes: A risk communication primer for patient decision aid developers. BMC Med Informatics Decis Making. 2013;13(Suppl 2):S7. 
19. Garcia-Retamero R, Galesic M. Who profits from visual aids: Overcoming challenges in people's understanding of risks. Social Science \& Medicine. 2010;70:1019-1025.

20. Garcia-Retamero R, Galesic M, Gigerenzer G. Do icon arrays help reduce denominator neglect? Med Decis Making. 2010;30:672-684.

21. National Vascular Disease Prevention Alliance (NVDPA). Guidelines for the management of absolute cardiovascular disease risk. Approved by the National Health and Medical Research Council (NHMRC), 2012. ISBN: 978-0-9872830-1-6.

22. Dillard AJ, Ferrer RA, Ubel PA, Fagerlin A. Risk perception measures' associations with behavior intentions, affect, and cognition following colon cancer screening messages. Health Psychol. 2012;31:106-113.

23. Cameron LD. Illness risk representations and motivations to engage in protective behavior: The case of skin cancer risk. Psychol Health. 2008;23:91-112.

24. Lee TJ, Cameron LD, Wunsche B, Stevens C. A randomized trial of computer-based communications using imagery and text information to alter representations of heart disease risk and motivate protective behaviour. Brit J Health Psychol. 2011;16:72-91.

25. Anderson K, Odell P, Wilson P, Kannel W. Cardiovascular disease risk profiles. Am Heart J. 1991;121:293-298.

26. Dunstan DW, Zimmet PZ, Welborn TA, Cameron AJ, Shaw J, de Courten M, et al. The Australian Diabetes, Obesity and Lifestyle Study (AusDiab): Methods and response rates. Diabetes Res Clin Pract. 2002;57:119-129.

27. Fagerlin A, Zikmund-Fisher BJ, Ubel PA, Jankovic A, Derry HA, Smith DM. Measuring numeracy without a math test: Development of the subjective numeracy scale. Med Decis Making. 2007; 27:672-680.

28. Armitage C, Conner M. Efficacy of the theory of planned behaviour: A meta-analytic review. Brit J Social Psychol. 2001;40:471-499. 
29. Francis J, Eccles M, Johnston M, Walker A, Grimshaw J, Foy R, et al. Constructing questionnaires based on the theory of planned behaviour: A manual for health services researchers. Newcastle upon Tyne, UK: Centre for Health Services Research, University of Newcastle upon Tyne. 2004.

30. Catellier JRA, Yang ZJ. Trust and affect: How do they impact risk information seeking in a health context? J Risk Res. 2012;15(8):897-911.

31. Scherer LD, Ubel PA, McClure J, Greene SM, Alford SH, Holtzman L, et al. Belief in numbers: When and why women disbelieve tailored breast cancer risk statistics. Patient Educ Couns. 2013;92(2):253-259.

32. Kievik M, ter Huurne EFJ, Gutteling JM. The action suited to the word? Use of the framework of risk information seeking to understand risk-related behaviors. J Risk Res. 2012;15:131-147.

33. Smith BJ, Marshall AL, Huang N. Screening for physical activity in family practice: Evaluation of two brief assessment tools. Am J Prev Med. 2005;29:256-264.

34. O'Hara BJ, Phongsavan P, Venugopal K, Eakin EG, Eggins D, Caterson H, et al. Effectiveness of Australia's Get Healthy Information and Coaching Service: Translational research with population wide impact. Prev Med. 2012;55(4):292-98.

35. Witteman HO, Fuhrel-Forbis A, Wijeysundera HC, Exe N, Dickson M, Holtzman L, et al. Animated randomness, avatars, movement, and personalization in risk graphics. J Med Internet Res. 2014;16(3):294-313 (Multimedia Appendix 3).

36. Bonner C, Newell BR. How to make a risk seem riskier: The ratio bias versus construal level theory. Judgm Decis Making. 2008;3:411-416.

37. Lopez-Gonzalez AA, Aguilo A, Frontera M, Bennasar-Veny M, Campos I, Vicente-Herrero T, et al. Effectiveness of the Heart Age tool for improving modifiable cardiovascular risk factors in a Southern European population: A randomized trial. Eur J Prev Cardiol. In press.

38. Harle CA, Downs JS, Padman R. A clustering approach to segmenting users of internet-based risk calculators. Methods Inf Med. 2011;50(3):244-252. 
Table 1. Participant characteristics and risk results by randomised risk format $(n=570)$

\begin{tabular}{|c|c|c|}
\hline & $\begin{array}{l}\text { Absolute risk } \\
\quad(n=281)\end{array}$ & $\begin{array}{c}\text { Heart age } \\
(n=289)\end{array}$ \\
\hline \multicolumn{3}{|l|}{ Demographic risk factors } \\
\hline Age, mean (SD) & $54(6)$ & $54(6)$ \\
\hline Male, $n(\%)$ & $134(48)$ & $153(53)$ \\
\hline Aboriginal or Torres Strait Islander, $n(\%)$ & $3(1)$ & $4(1)$ \\
\hline Numeracy, median (IQR) & $\begin{array}{c}4.3 \\
(3.4-5.0)\end{array}$ & $\begin{array}{c}4.4 \\
(3.6-5.0)\end{array}$ \\
\hline \multicolumn{3}{|l|}{ Lifestyle risk factors } \\
\hline \multicolumn{3}{|l|}{ Body mass index (pre-intervention), $n(\%)$} \\
\hline Underweight & $10(4)$ & $12(4)$ \\
\hline Normal & $95(34)$ & $106(37)$ \\
\hline Overweight & $92(33)$ & $99(34)$ \\
\hline Obese & $81(29)$ & $70(25)$ \\
\hline Cigarettes per day (pre-intervention), median (IQR) & $\begin{array}{c}69(25) \\
15 \\
(10-20)\end{array}$ & $\begin{array}{c}72(25) \\
15 \\
(10-25)\end{array}$ \\
\hline Adequate $\operatorname{diet}^{*}$ (pre-intervention), $n(\%)$ & $50(18)$ & $51(18)$ \\
\hline Adequate physical activity $^{\dagger}$ (pre-intervention), $n(\%)$ & $124(44)$ & $128(44)$ \\
\hline \multicolumn{3}{|l|}{ Clinical risk factors } \\
\hline BP assessed in last year, $n$ (\%) & $207(74)$ & $197(68)$ \\
\hline Told BP raised by health professional, $n(\%)$ & $45(16)$ & $42(15)$ \\
\hline Cholesterol assessed in last year, $n(\%)$ & $144(51)$ & $142(49)$ \\
\hline Told cholesterol raised by health professional, $n(\%)$ & $44(16)$ & $61(21)$ \\
\hline Systolic BP (value/estimate), mean (SD) & $122(11)$ & $121(9)$ \\
\hline Cholesterol ratio (value/estimate), mean (SD) & $3.8(0.7)$ & $3.8(0.7)$ \\
\hline \multicolumn{3}{|l|}{ Calculated risk results } \\
\hline 5-year absolute CVD risk, mean (SD) & $4.3(3.1)$ & $4.3(3.0)$ \\
\hline \multicolumn{3}{|l|}{ 5-year absolute CVD risk category, $n(\%)$} \\
\hline Low risk $(<10 \%)$ & $266(95)$ & $269(93)$ \\
\hline Moderate risk (10-15\%) & $14(5)$ & $20(7)$ \\
\hline High risk (>15\%) & $1(0)$ & $0(0)$ \\
\hline Heart age, mean (SD) & $57(11)$ & $57(10)$ \\
\hline Lower heart age than current age, $n$ (\%) & $107(38)$ & $100(35)$ \\
\hline Same heart age as current age, $n(\%)$ & $3(1)$ & $4(1)$ \\
\hline Higher heart age than current age, $n(\%)$ & $171(61)$ & $185(64)$ \\
\hline
\end{tabular}

${ }^{*} \geq 2$ servings of fruit and $\geq 5$ servings of vegetable per day, in the last week; ${ }^{\dagger} \geq 3$ vigorous sessions, or $\geq 5$ moderate sessions, or 1-2 vigorous sessions plus 3-4 moderate sessions, in the last week IQR: interquartile range; BP: blood pressure; CVD: cardiovascular disease 


\begin{tabular}{|c|c|c|c|}
\hline & $\begin{array}{c}\text { Absolute } \\
\text { risk } \\
(n=281)\end{array}$ & $\begin{array}{l}\text { Heart age } \\
(n=289)\end{array}$ & p-value \\
\hline \multicolumn{4}{|l|}{ Intention outcomes (post-intervention) } \\
\hline To reduce smoking ${ }^{*}$, median (IQR) & $\begin{array}{c}4.7 \\
(3.7-6.0)\end{array}$ & $\begin{array}{c}5.0 \\
(4.0-5.7)\end{array}$ & $0.67^{* *}$ \\
\hline To improve diet ${ }^{*}$, median (IQR) & $\begin{array}{c}4.3 \\
(3.0-5.7)\end{array}$ & $\begin{array}{c}4.0 \\
(3.0-5.0)\end{array}$ & $0.47^{* *}$ \\
\hline To improve physical activity ${ }^{*}$, median (IQR) & $\begin{array}{c}4.3 \\
(3.5-5.7)\end{array}$ & $\begin{array}{c}4.3 \\
(3.7-5.3)\end{array}$ & $0.72^{* *}$ \\
\hline To improve diet/PA or diet/PA/smoking ${ }^{\dagger}$, median (IQR) & $\begin{array}{c}4.3 \\
(3.5-5.5)\end{array}$ & $\begin{array}{c}4.4 \\
(3.5-5.2)\end{array}$ & $0.72^{* *}$ \\
\hline To see GP for CVD risk assessment ${ }^{*}$, median (IQR) & $\begin{array}{c}2.0 \\
(1.0-3.0) \\
\end{array}$ & $\begin{array}{c}2.0 \\
(1.0-3.3) \\
\end{array}$ & $0.35^{* *}$ \\
\hline \multicolumn{4}{|l|}{ Psychological outcomes } \\
\hline \multicolumn{4}{|l|}{ Risk perception (post-intervention) } \\
\hline Results indicate low risk of heart attack/stroke, $n(\%)$ & $216(77)$ & $154(53)$ & \\
\hline Results indicate moderate risk of heart attack/stroke, $n$ (\%) & $60(21)$ & $121(42)$ & $<0.001^{\dagger+}$ \\
\hline Results indicate high risk of heart attack/stroke, $n(\%)$ & $5(2)$ & $14(5)$ & \\
\hline Correct recall (post-intervention), $n$ (\%) & $173(62)$ & $195(67)$ & $0.17^{+\dagger}$ \\
\hline Correct recall (after 2 weeks), $n$ (\%) & $37(16)$ & $77(32)$ & $<0.001^{\dagger+}$ \\
\hline Perceived credibility of results ${ }^{\ddagger}$ (post-intervention), median (IQR) & $\begin{array}{c}4.8 \\
(4.0-6.0)\end{array}$ & $\begin{array}{c}4.3 \\
(3.3-5.3)\end{array}$ & $<0.001^{* *}$ \\
\hline Positive emotional response ${ }^{\S}$ (post-intervention), median (IQR) & $\begin{array}{c}6.0 \\
(5.0-8.0)\end{array}$ & $\begin{array}{c}5.0 \\
(4.0-7.0)\end{array}$ & $<0.001^{* *}$ \\
\hline Negative emotional response ${ }^{\S}$ (post-intervention), median (IQR) & $\begin{array}{c}2.3 \\
(1.0-4.7) \\
\end{array}$ & $\begin{array}{c}3.0 \\
(1.0-5.0) \\
\end{array}$ & $0.40^{* *}$ \\
\hline \multicolumn{4}{|l|}{ Behavioural outcomes } \\
\hline Information seeking (post-intervention), $n$ (\%) & $19(7)$ & $15(5)$ & $0.43^{++}$ \\
\hline Smoker (after 2 weeks), $n$ (\%) & $58(25)$ & $55(23)$ & $0.62^{+\dagger}$ \\
\hline Cigarettes per day (after 2 weeks), median (IQR) & $\begin{array}{c}12 \\
(6-20)\end{array}$ & $\begin{array}{c}10 \\
(3-20)\end{array}$ & $0.72^{* *}$ \\
\hline Adequate diet "I (after 2 weeks), $n$ (\%) & $27(12)$ & $22(9)$ & $0.45^{\dagger+}$ \\
\hline Adequate physical activity" (after 2 weeks), $n$ (\%) & $91(40)$ & $100(42)$ & $0.74^{+\dagger}$ \\
\hline Made GP appointment (after 2 weeks), $n$ (\%) & $23(10)$ & $27(11)$ & $0.78^{+\dagger}$ \\
\hline
\end{tabular}

${ }^{*}$ Average of 3 items on 1-7 Likert scale (1=strongly disagree, $7=$ strongly agree); ${ }^{\dagger}$ Average of 6/9 items on 1-7 Likert scale depending on smoking status ( $1=$ strongly disagree, $7=$ strongly agree); ${ }^{\ddagger}$ Average of 4 items on $1-7$ Likert scale ( $1=$ strongly disagree, $7=$ strongly agree); ${ }^{\S}$ Average of 3 items on $0-10$ Likert scale $(0=$ none of this feeling, $10=a$ lot of this feeling); $" 1 \geq 2$ servings of fruit and $\geq 5$ servings of vegetable per day in the last week; ${ }^{q} \geq 3$ vigorous sessions, or $\geq 5$ moderate sessions, or 1-2 vigorous sessions plus 3-4 moderate sessions, in the last week; higher scores indicate more of attribute.

${ }^{* *}$ Mann-Whitney test, ${ }^{+\dagger}$ Chi-square test

IQR: Interquartile range; PA: physical activity; GP: general practitioner; CVD: cardiovascular disease. 